\title{
Determination of Selected Polycyclic Aromatic Compounds in Particulate Matter Samples with Low Mass Loading: An Approach to Test Method Accuracy
}

\author{
Susana García-Alonso, ${ }^{1}$ Rosa María Pérez-Pastor, ${ }^{1}$ Víctor Archilla-Prat, ${ }^{2}$ \\ David Sanz-Rivera, ${ }^{3}$ Enrique Rojas-García, ${ }^{3}$ \\ Gaizka Aragón-Urrengoetxea, ${ }^{2}$ and Jesús Javier Rodríguez-Maroto ${ }^{3}$ \\ ${ }^{1}$ CIEMAT, Technology Department, Madrid, Spain \\ ${ }^{2}$ INTA, Madrid, Spain \\ ${ }^{3}$ CIEMAT, Environment Department, Madrid, Spain
}

Correspondence should be addressed to Susana García-Alonso; susana.garcia@ciemat.es

Received 26 February 2017; Revised 19 May 2017; Accepted 11 June 2017; Published 27 July 2017

Academic Editor: Kamlesh Shrivas

Copyright (C) 2017 Susana García-Alonso et al. This is an open access article distributed under the Creative Commons Attribution License, which permits unrestricted use, distribution, and reproduction in any medium, provided the original work is properly cited.

\begin{abstract}
A miniaturized analytical procedure to determine selected polycyclic aromatic compounds (PACs) in low mass loadings ( $<10 \mathrm{mg}$ ) of particulate matter (PM) is evaluated. The proposed method is based on a simple sonication/agitation method using small amounts of solvent for extraction. The use of a reduced sample size of particulate matter is often limiting for allowing the quantification of analytes. This also leads to the need for changing analytical procedures and evaluating its performance. The trueness and precision of the proposed method were tested using ambient air samples. Analytical results from the proposed method were compared with those of pressurized liquid and microwave extractions. Selected PACs (polycyclic aromatic hydrocarbons (PAHs) and nitro polycyclic aromatic hydrocarbons (NPAHs)) were determined by liquid chromatography with fluorescence detection (HPLC/FD). Taking results from pressurized liquid extractions as reference values, recovery rates of sonication/agitation method were over $80 \%$ for the most abundant PAHs. Recovery rates of selected NPAHs were lower. Enhanced rates were obtained when methanol was used as a modifier. Intermediate precision was estimated by data comparison from two mathematical approaches: normalized difference data and pooled relative deviations. Intermediate precision was in the range of $10-20 \%$. The effectiveness of the proposed method was evaluated in PM aerosol samples collected with very low mass loadings $(<0.2 \mathrm{mg})$ during characterization studies from turbofan engine exhausts.
\end{abstract}

\section{Introduction}

There is an increasing concern about the chemical characterization of environmental samples of size-segregated samples [1-3]. This leads to the analysis of reduced sample sizes of particulate matter (PM) containing very low levels of pollutants. These samples require improving both analytical method extraction and quality. Among chemicals typically linked to PM, PACs, a well-known family of toxic contaminants, are of great concern. Conventional analytical procedures usually require samples with mass loadings in order of dozens of mg (i.e., ambient air samples collected by using high volume sampler during 24 hours). Among extraction techniques, soxhlet (SX), microwave assisted extraction (MAE), pressurized liquid extraction (PLE), and ultrasonic extractions (US) have been extensively used $[4,5]$. Large amount of solvent (SX), high cost (PLE and MAE), and low extraction efficiency (US) are important limitations when large sets of samples with reduced mass loadings are considered for analysis.

Extraction of PM samples with small mass loadings has been carried out using PLE $[1,2]$ and thermal desorption $[6,7]$. These extractions are expensive in instrument and maintenance. Due to lower equipment cost and ease of operation, extraction by US stands as an attractive alternative 
$[3,8]$. Hence, procedures based on reducing sample size and solvent requirements have been developed to achieve a miniaturization strategy for US extraction [9-11]. When the analyses of samples with low mass loadings are discussed, the validation of analytical method is an essential issue. Not enough attention is always paid to this matter.

A validation study includes the estimation of the accuracy of analytical method, which reflects the closeness of a measured value to a true value. Therefore, two components of accuracy must be assessed: trueness and precision [12]. If intralaboratory tests have to be carried out, the performance of proposed method will be based on the use of recovery assessments, reference materials, or comparison with a reference method [13]. The comparison of results obtained by proposed method with those found with a comparison method of known accuracy is a useful tool for this kind of studies. Perhaps a conclusion of quantitative extraction after the analysis of certified reference materials could not be extended at real samples. Possible matrix effects coming from the sample material could be easily delimiting the quality of final results.

As a part of aerosol characterization studies in gas turbine exhaust [12], PAC determination in PM samples with low mass loadings was required. In the present study, an affordable procedure based on an extraction by sonication and agitation with small volumes of solvent was evaluated. Quality of results was pursued by means of the evaluation of the extraction efficiency and intermediate precision from the analyses of PM samples. Keeping simplicity and low cost as advantages, our main goal was to evaluate analytical procedure for determination of selected PACs using real samples. This is rarely applied. Moreover, this work demonstrates the application of the proposed method.

The applicability of the method was tested at the most disadvantageous case: PM samples with very low mass loadings (in order of micrograms) and PAC levels close to analytical detection limits (in order up to 1 ng per entire filter). These extremely low levels of PM and analyte require an assessment of the reliability of analytical measurements.

\section{Materials and Methods}

2.1. Reagents and Standards. Acetic acid (for analytical grade), dichloromethane (for organic trace analysis), and acetonitrile (HPLC grade) from SDS, Carlo Erba (Val de Reuil, France), were used for sample preparation and chromatographic analysis. Sodium borohydride (99\%) and copper(II) chloride (97\%) from Sigma Aldrich (Steinheim, Germany) were used for derivatization reaction to determine NPAHs.

The PAH Mix 9 calibration check solutions $\left(10 \mu \mathrm{g} \mathrm{mL}^{-1}\right.$ in acetonitrile) were supplied by Dr. Ehrenstorfer GmbH (Augsburg, Germany), containing naphthalene $(\mathrm{Na})$, acenaphthene (Ace), fluorene (F), phenanthrene (Ph), anthracene (An), fluoranthene $(\mathrm{Fl})$, pyrene $(\mathrm{Pyr})$, benz [a] anthracene $(\mathrm{BaA})$, chrysene (Cry), benz[b]fluoranthene (BbF), benz[k]fluoranthene $(\mathrm{BkF})$, benz[a]pyrene $(\mathrm{BaP})$, dibenz $[\mathrm{a}, \mathrm{h}]$ anthracene $(\mathrm{DBA})$, and benzo[g,h,i]perylene (BghiP). 1-Methyl-naphthalene (1$\mathrm{MeNa}), 98.6 \%$, and 2-methyl-naphthalene (2-MeNa), 97.2\%, were purchased from Ultra Scientific (North Kingstown, USA).

Individual nitro-PAH (NPAHs) calibration check solutions (methanol, $10 \mu \mathrm{g} / \mathrm{mL}$ ) were supplied by Dr. Ehrenstorfer Reference Materials (Augsburg, Germany). Selected NPAHs were 2-nitro-naphthalene (2-NNa), 3-nitro-phenanthrene (3NPhe), 9-nitro-phenanthrene (9-NPhe), 1-nitro-pyrene (1$\mathrm{NPyr}$ ), and 3-nitro-fluoranthene (3-NFl). Stock solutions prepared in methanol were used to identify and quantify the aromatic compounds.

The SRM 1649b, an urban dust, was obtained from the National Institute of Standards and Technology (NIST, USA).

2.2. Samples and Preparation. For the evaluation of the extraction efficiency and intermediate precision of results from the proposed method, twelve ambient air PM10 samples were taken and analyzed. Sampling site was the National Institute for Aerospace Technology (INTA), located at $20 \mathrm{~km}$ Northeast of Madrid city. A high volume MCV CAV-Amb sampler loaded with Whatman glass fiber filters $(102 \mathrm{~mm}$ diameter) was used. Filter samples were wrapped separately in aluminum foil and stored in a freezer at $-20^{\circ} \mathrm{C}$ until analysis, within 1 week from collection.

The ambient air samples were taken with mass loadings of particulate matter between 3 and $40 \mathrm{mg}$ as follows. Sampling was carried out during 24 hours $\left(30 \mathrm{~m}^{3} \mathrm{~h}^{-1}\right)$ for consecutive days between April and November of 2014. Only samples 10, 11 , and 12 were collected during $30 \mathrm{~min}, 50 \mathrm{~min}$, and 2 hours to achieve lower mass loadings of particulate matter $(3 \mathrm{mg}$, $5 \mathrm{mg}$, and $11 \mathrm{mg}$, resp.). All filters were accurately weighed before and after sampling at constant humidity to a precision of $10 \mu \mathrm{g}$ prior to sampling.

Each sample was divided into four subsamples according to the flow diagram presented in Figures $1 \mathrm{~s}$ and $2 \mathrm{~s}$ of the Electronic Supplementary Material (ESM), available online at https://doi.org/10.1155/2017/3517457. Hence, four subsamples of each PM10 sample were weighed, treated, and analyzed separately to evaluate four extraction procedures based on the following:

(i) MAE: PM subsamples were extracted using an Ethos Sel (Millestone) at $900 \mathrm{w}\left(120^{\circ} \mathrm{C}\right)$, during $40 \mathrm{~min}$. Dichloromethane was used as extraction solvent $(15 \mathrm{~mL})$.

(ii) PLE: PM subsamples were extracted using an ASE350 (Dionex Corporation) and dichloromethane (DCM) as extraction solvent $\left(100^{\circ} \mathrm{C}, 10.34 \mathrm{psi}\right.$ and 1 static cycle). The total volume of extract which was obtained after extraction corresponded to $55 \mathrm{~mL}$.

(iii) $A G-D$ and $A G-D M$ : PM subsamples were extracted in closed tubes containing $5 \mathrm{~mL}$ of extraction solvent (dichloromethane and dichloromethane/methanol $2: 1$, resp.). The extraction was performed in an ultrasonic bath for $15 \mathrm{~min}$, using a Selecta Ultrasons$\mathrm{H}$ (Barcelona, Spain). Subsequently the tubes were agitated for short time using an automatic shaker with a touch function (IKA vortex 1, Staufen, Germany). After the main extraction, additional extractions were 
carried out by adding $1 \mathrm{~mL}$ of extraction solvent (twice) and agitating for a short time. All extracts were then combined.

The final extracts were filtered $(0.22 \mu \mathrm{m}$ pore size PTFE) and slowly concentrated in a drying step by stream of nitrogen. Finally, dried samples were redissolved by adding $150-250 \mu \mathrm{L}$ of methanol for PAH determination.

Once selected PAHs were quantified; derivatization reaction of extracts was performed for NPAH determination [14]. The procedure was based on addition of $0.5 \mathrm{~mL}$ of $0.05 \%$ aqueous solution of $\mathrm{CuCl}_{2}$ and $0.05 \mathrm{~g}$ of $\mathrm{NaBH}_{4}$ to $0.5 \mathrm{~mL}$ of extract in methanol. This mixture was maintained at room temperature for $1 \mathrm{~h}$. After this, $0.5 \mathrm{~mL}$ of water and $1 \mathrm{~mL}$ of dichloromethane were added and then agitated for extraction of amino-PAHs. Dichloromethane extract was collected with a syringe and two reextractions were performed to ensure recovery. Combined extracts were concentrated under nitrogen flow to change solvent (acetonitrile, $100 \mu \mathrm{L}$ ).

The applicability of the method was then tested from the analyses of PM aerosols at very low mass loadings. Involved samples corresponded to the following:

(i) Mass loadings of $0.2 \mathrm{mg}$ collected on quartz fiber filter $(47 \mathrm{~mm})$ by means of a Low Pressure Impactor

(ii) Mass loadings of $0.02 \mathrm{mg}$ collected on aluminum (Al) foils $(47 \mathrm{~mm})$ by means of a holder filter

These samples were treated by AG-D method and analyzed as previously indicated.

2.3. Chromatographic Analysis. Sample analyses were performed on an Agilent series 1200 liquid chromatograph coupled to an Agilent 1100 fluorescent detector (Waldbronn, Germany). Particular conditions were previously optimized and well established [14]. Briefly, a supelcosil C18 column (Supelco, $250 * 4.6 \mathrm{~mm}, 5 \mu \mathrm{m}$ ) and an eclipse XDB C18 column (Agilent, $150 * 4.6 \mathrm{~mm}, 5 \mu \mathrm{m}$ ) were used for PAH and NPAH determinations, respectively. The mobile phase rates were $1.5 \mathrm{~mL}$ and $1.0 \mathrm{~mL}$ for PAH and NPAH determinations, respectively. Operational conditions of HPLC/FD are compiled in Table 1s (ESM).

Quantification was performed by external standards with five-point calibration. Linear response was established from $10 \mathrm{ng} \mathrm{mL}^{-1}$ to $1000 \mathrm{ng} \mathrm{mL}^{-1}$ for PAHs. Due to the lower detector sensitivity for NPAHs, its linear concentration range was from $100 \mathrm{ng} \mathrm{mL}^{-1}$ to $1000 \mathrm{ng} \mathrm{mL}^{-1}$. Regression coefficients were above 0.999 . Analyte concentration should be in the middle of the calibration range of standards, but a response factor based on a lower/higher point of standard solutions was applied if this was not possible [15].

\section{Results and Discussion}

3.1. Quality Control. Instrumental limit of detection of each PAC was calculated as twice the standard deviations deduced from analyses of three standard solutions containing $1 \mathrm{ng} \mathrm{mL}^{-1}$ and $10 \mathrm{ng} \mathrm{mL}^{-1}$ for selected PAHs and NPAH, respectively. Derived values were between $0.5 \mathrm{ng} \mathrm{mL}^{-1}$ and $5 \mathrm{ng} \mathrm{mL}^{-1}$ for selected PAHs and NPAHs, respectively.
The analysis of blanks (i.e., chemical analysis excluding PM samples) indicated the potential cross contamination for the more volatile PAHs if extreme care is not taken. Due to ubiquitous character of PAH compounds in the air, laboratory inventory, and low volumes of extracts, there is a risk of contamination. Therefore, to determine blank contents for each set of sample analyses is recommended. The very low levels require a rigorous protocol to ensure minimal sample contamination from vials, syringes, solvents, and drying step.

Blank filters of glass fiber, quartz, and aluminum were handled in the same way as the samples by agitation procedure and these trials concluded to detectable contents only for the more volatile PAHs. In detail, 1-methyl-naphthalene, 2methyl-naphthalene, and phenanthrene were associated with increases more than 10 times the limit value considered instrumental. The detection limits were set as twice the standard deviation of the measured values.

Following a more conventional protocol for testing accuracy of the proposed method, the reference material SRM 1649b from the National Institute of Standards and Technology of USA (NIST) was selected for initial comparisons. The reference material SRM 1649b provides typical urban dust matrix for evaluating the reliability of analytical methods. Four aliquots of $10 \mathrm{mg}$ were subject to analytical protocol by agitation using dichloromethane as solvent. PAH mass fraction values were in the range of those certified (Table 2s, ESM), except for benzo[a]pyrene and dibenzo[ah] anthracene, with lower recoveries (close to 70\%). However, the recovery values were considered acceptable according to the published acceptable recovery percentages as a function of the analyte concentration [12].

Regarding NPAH results, only 1-nitro pyrene could be compared and a recovery of $80 \%$ was reached.

3.2. Measured PACs and Test for Assessing Trueness. The study for comparison among the investigated extraction methods was carried out to evaluate the performance of the developed procedure by agitation/sonication. In particular, ambient air samples were divided into four subsamples, weighed, and then considered as independent or parallel subsamples for analysis, as previously indicated.

Measured PAC masses (in ng) per subsample obtained from the investigated extraction methods are compiled in Table 1. The sum of masses for selected PAHs and NPAHs ranged from $24 \mathrm{ng}$ to $800 \mathrm{ng}$ and $5.5 \mathrm{ng}$ to $240 \mathrm{ng}$, respectively. The individual quantities of most abundant PAHs varied between 1 and $150 \mathrm{ng}$ and together they accounted for $75 \%$ of total mass. The most abundant PAHs were 2-MeNa, $\mathrm{Ph}$, Fl, Pyr, Cry, BbF, and BghiP. Ace and F were not detected. Regarding selected NPAHs, 9-NPh and 1-NPyr were the most abundant, which ranged from $1.2 \mathrm{ng}$ to $100 \mathrm{ng}$.

For assessing trueness of AG-D method, ambient air samples 1-5 were divided and considered as independent subsamples for extraction by MAE, PLE, AG-D, and AG-DM methods. Mass aerosol loadings per subsample were about $40 \mathrm{mg}$ (samples 2-5) and $16 \mathrm{mg}$ (sample 1), so selected PACs were determined at around $10 \mathrm{mg}$ per subsample and $4 \mathrm{mg}$ per subsample after cutting it (Table 1). 
荇|

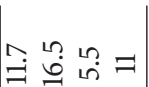
장

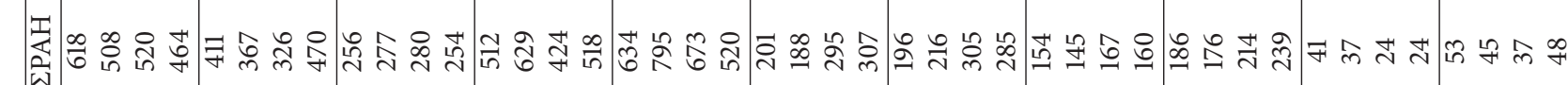
焉密

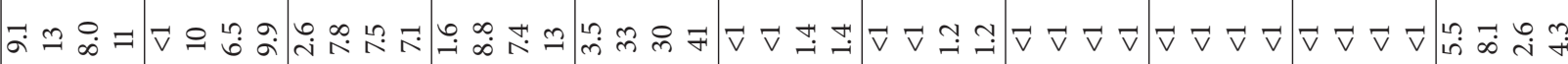

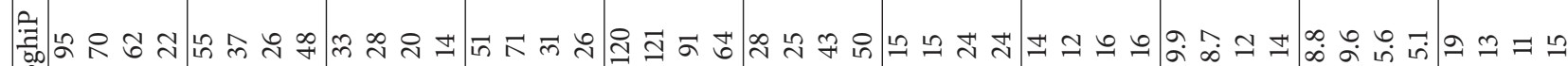

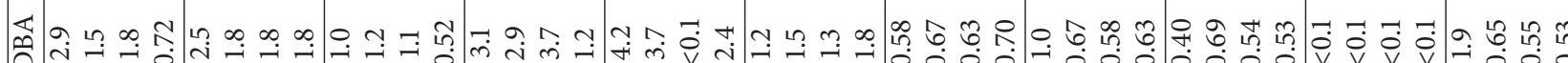

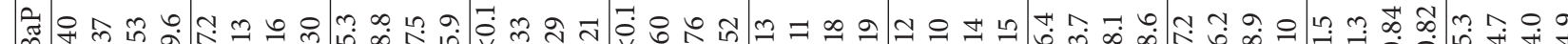

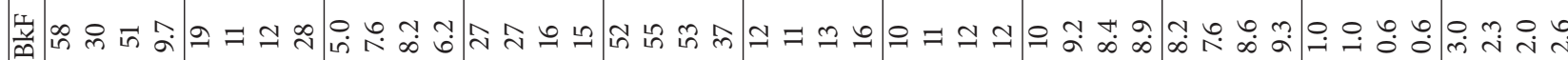

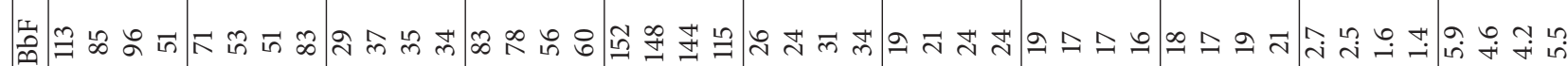

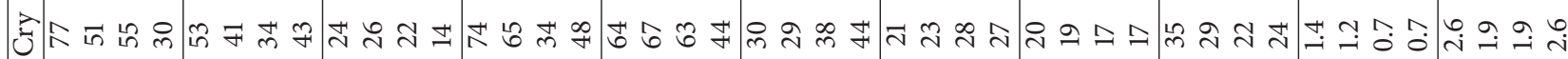

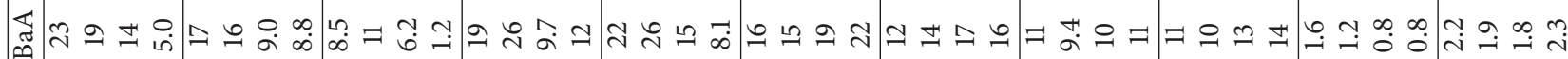

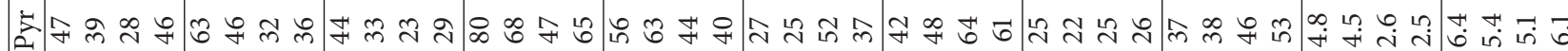

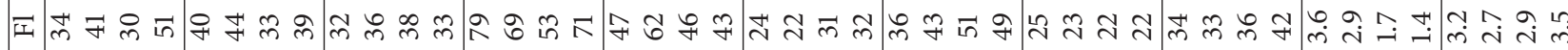

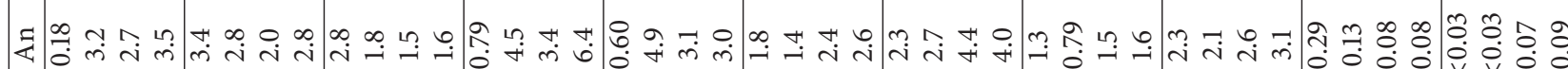

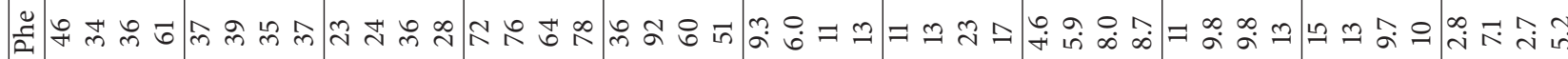

艺に

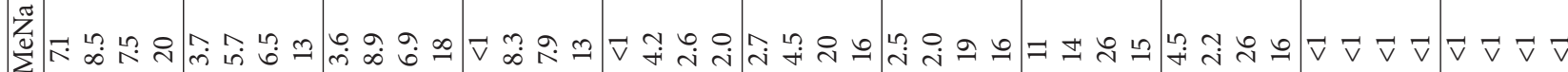

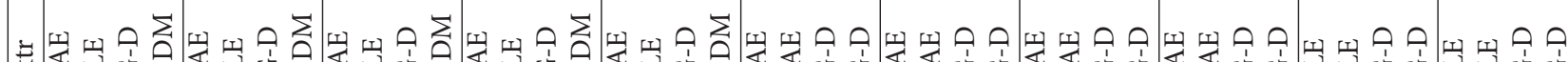




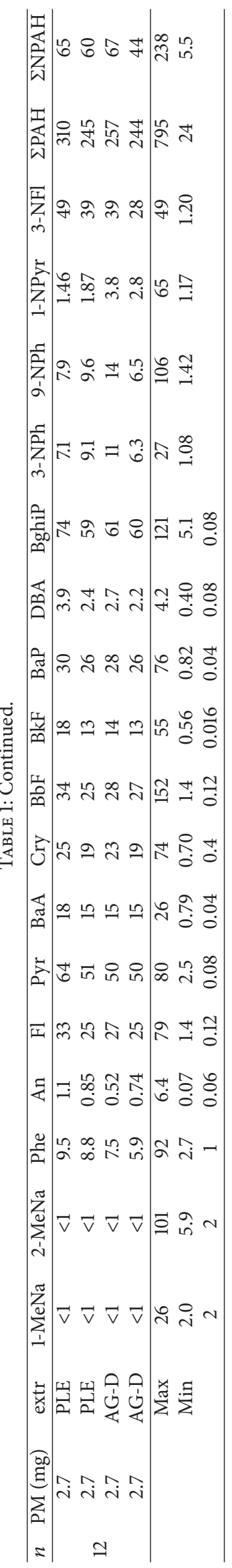





Figure 1: Mean values (ng per subsample) of selected PACs measured studied extraction methods. The abbreviations for PACs and extraction procedures are expanded in Reagents and Standards and Samples and Preparation, respectively.

The experiment was designed as follows: the first subsample was extracted by MAE, the second one by PLE, the third by AG-D, and the last one by AG-DM. A preliminary comparison was performed by grouping results by extraction technique and deducing mean of measured masses (Figure 1). As can be seen, results were in good agreement. In contrast to results obtained from SRM 1649 analyses, no differences were observed among investigated methods for benzo[a]pyrene and dibenzo[ah]anthracene measurements. When applying the agitation method using a mix of dichloromethane/methanol as solvent, high levels of the more volatile PAHs were obtained, while heavier PAHs (BaA, Cry, $\mathrm{BkF}, \mathrm{BaP}$, and $\mathrm{BghiP}$ ) provided lower response. This is coherent to the more polar character of solvent and explains the difficulty for extracting the heavier and less polar PAHs. Similarly, NPAHs (more polar) corresponded to higher levels using methanol as modifier for agitation extraction.

Taking PLE results as reference values, we considered testing proposed method as the closeness of agreement between both AG-D and PLE results [16, 17]. In detail, the calculation of recoveries $\left(R_{i}\right)$ was performed by computing the individual ratios for each PAC according to

$$
R_{i}=\frac{x_{\mathrm{AGD}}}{x_{\mathrm{PLE}}}
$$

where $x_{\mathrm{AGD}}$ and $x_{\mathrm{PLE}}$ are the mass of quantified PAC (ng) after agitation/sonication and pressurized liquid extractions using dichloromethane as solvent, respectively. Results obtained from the analyses of samples 1-5 and 9-11 were used for calculation. Quantified mass of selected PAC were between 1 and $75 \mathrm{ng}$ (Table 1 ).

In order to detect outlier values, Grubb's test was applied to each data set. To verify whether the minimum or the maximum value was acceptable, the ratio $G_{i}$ was calculated according to

$$
G_{i}=\frac{\left|R_{m / M}-\bar{R}_{i}\right|}{\mathrm{sd}}
$$

where $R_{m / M}$ denotes the maximum or minimum ratio value, $\bar{R}_{i}$ is the mean ratio, and sd refers to the standard deviation of data set. The value from this equation was compared with two-tailed critical $t_{\mathrm{tab}}$, for $(n-1)$ degrees of freedom at $95 \%$ confidence (where $n$ is the number of values). When $G_{i}$ values were higher than $t_{\mathrm{tab}}$, they were rejected and a recalculation was carried out. Of the data set, 4 outliers were discarded. Final results are included in Table 2. Mean values were very acceptable for PAHs (>80\%). But lower recoveries were obtained for the less abundant PAHs and NPAHs.

A test of significance was then used for verifying if the ratio between the value of the mean of recoveries $\bar{R}_{i}$ and its relative standard deviation (sd) was significantly different. The average recovery was tested for significance by using the Student's $t$-test:

$$
t=\frac{\bar{R}_{i}-1}{s_{\text {rec }} / \sqrt{n}}
$$

If the obtained $t$ value is lower than the tabulated value for $(n-$ 1) degrees of freedom at $95 \%$ significance level, we consider the results from PLE and AG-D methods comparable for PAC measurements. Only Pyr and BghiP corresponded to slightly lower values from the proposed method. However, no significant differences were found (Table 2).

Regarding NPAH results, lower ratios were found, especially for 3-NPhe. The low mass levels together with the low mass loading are critical factors to the relatively low sensitivity of the derivatization method proposed for HPLC/FD analysis. These limitations should be improved by extraction with a mix of dichloromethane/methanol as previously mentioned.

Taking into account the fact that NPAHs are minor contributors and the higher interest in quantifying less volatile PAHs, dichloromethane was chosen.

3.3. Estimation of the Intermediate Precision. From a more practical point of view, our goals were focused on reporting a range where results can be expected rather than detailed analyses of uncertainty sources. On this basis, intermediate precision was investigated by comparing results among the selected extraction methods and different PM mass loadings. In detail, seven ambient air samples were taken (samples 6-12) and each one was divided into four subsamples to analyze by pairs as follows (Figure 2S, ESM).

(i) Comparison between MAE and AG-D procedures: Four samples with PM mass loadings between 5 


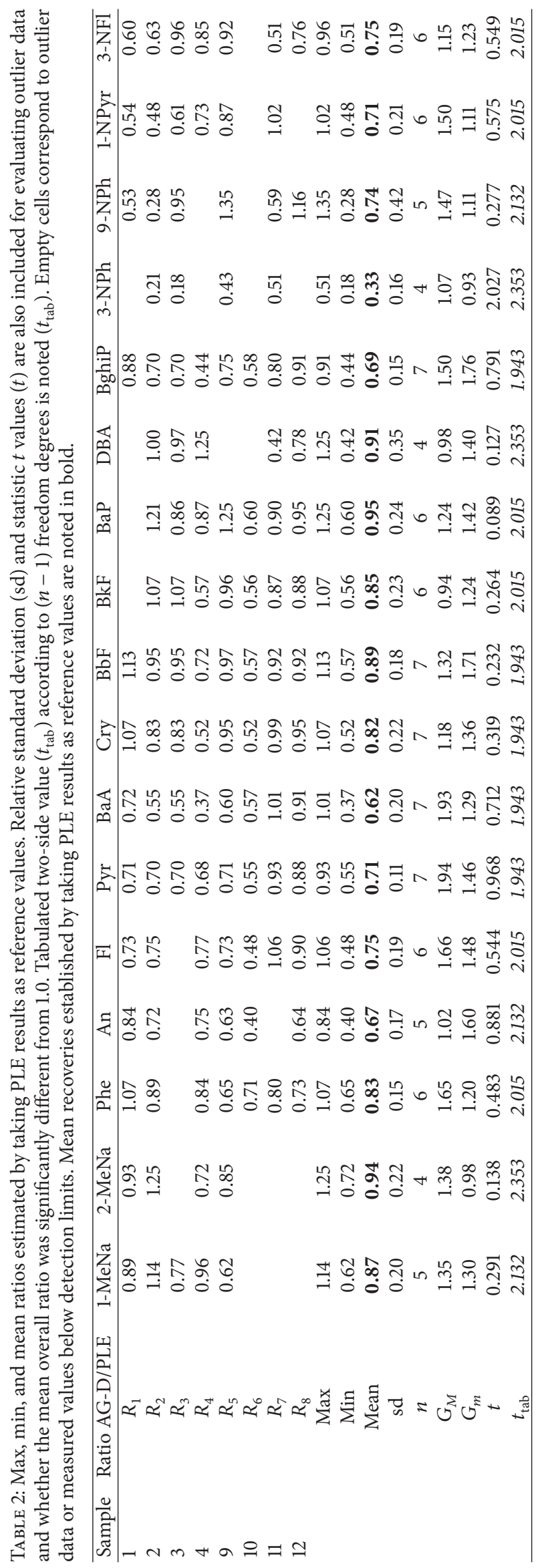


and $8 \mathrm{mg}$ were divided and analyzed by pairs in different days. In particular, results corresponded to the analyses of samples 5, 6, 7, and 8 (Table 3).

(ii) Comparison between PLE and AG-D procedures: Analogously, three ambient air samples with lower PM mass loadings (0.7-2.8 mg) were divided and analyzed by pairs in different days. These low amounts of PM were chosen to investigate the scope of the developed method. In particular, results corresponded to the analyses of samples 9, 10, and 11 (Table 3).

Once data compiled, intermediate precision was calculated based on two practical approaches for a better estimation:

(1) Percentage difference: by estimating a relative standard deviation of the normalized differences (Example A4, EURACHEM [18])

(2) $\mathrm{RSD}_{\text {pooled }}$ : by calculating a pooled relative deviation

Namely, the precision was estimated over a time period, using the standard deviation of pairs of results. The analyses corresponded to 7 samples analyzed 4 times over a period of time.

3.3.1. Percentage Difference. The calculation was carried out by grouping results in pairs, according to each filter sample and extraction technique (Table $3 \mathrm{~s}, \mathrm{ESM}$ ). The normalized difference data was then calculated as the difference divided by mean value for each pair of data. These results really show a difference between two values of each pair as a percentage of both values. Therefore, they provide a measure of the overall variability between analyses. On this basis, calculating the standard deviation of the normalized differences gives an estimation of relative standard uncertainty due to run-to-run variation of the overall analytical process.

3.3.2. $R S D_{\text {pooled }}$. Results in pairs, according to the duplicate analyses of each sample and extraction technique, were grouped to deduce relative standard deviations. The mean relative standard deviation $\left(\mathrm{RSD}_{\text {pooled }}\right)$ was then expressed according to

$$
\mathrm{RSD}_{\text {pooled }}=\sqrt{\frac{\sum \operatorname{rsd}_{i}^{2}\left(n_{i}-1\right)}{\sum\left(n_{i}-1\right)}},
$$

where $\operatorname{rsd}_{i}$ corresponds to relative standard deviation deduced from each ambient air sample, $i$, in duplicate.

The results of intermediate precision obtained are compiled in Table 3. Empty cells corresponded to values below detection limits. Also the relative deviations derived from previous work have been included [14]. It must be noted that these results were obtained from the analysis of a single sample after cutting four subsamples, which were treated and analyzed as independent samples. Deviation values deduced with this approach were slightly higher. Measures from a sole ambient air sample can lead to implying an overestimation of the analytical variability by mathematical calculation itself.
In general, good concordance was found between results obtained from both mathematical approaches.

As can be seen, $\mathrm{RSD}_{\text {pooled }}$ corresponding to agitation results were generally below $10 \%$ for PAH and 9-NPhe with masses above $10 \mathrm{ng}$ (subsamples with PM mass loadings between 5 and $8 \mathrm{mg}$, Table 3). Higher relative standard deviation was found (10-15\%) when mass levels were between 1 and $5 \mathrm{ng}$ (subsamples with PM mass loadings between 0.7 and $2.8 \mathrm{mg}$, Table 3 ). In the case of selected NPAHs, only 9NPhe was quantified and no enough data were obtained for the rest of investigated NPAHs. For microwave method, lower values and increases of variability (up to 50\%) were found for the isomers of methyl naphthalene, the most volatile. These observations likely correspond to losses due to the applied heat and the more volatile nature of these compounds.

For NPAHs, intermediate deviation values were higher, around $20 \%$, which is justified by the increase of experimental variability associated with additional derivatization step for chromatographic analysis. Calculations could be performed when higher NPAH mass levels were measured (subsamples with PM mass loadings between 5 and $8 \mathrm{mg}$, Table 3). PLE shows a slightly lower variability of the results. This is in agreement with the higher efficiency of PLE extraction and difficulty of the extraction of several PACs from particulate matter [19].

\subsection{Application to Real PM Samples with Very Low Mass} Loadings. As a part of characterization studies from the turbofan engine exhaust [20], aerosols on quartz fiber filter and aluminum ( $\mathrm{Al})$ foils $(47 \mathrm{~mm})$ were collected by means of a Low Pressure Impactor and a filter holder, respectively. Measurements were focused on preliminary studies on particle size distributions of PM. Exhaust PM sampling was conducted to explore detection limits of analysis and optimize parameters such as time and flow at different engine conditions. This is often a limiting factor to obtain sufficient sample mass for analysis. In detail, the effectiveness of the proposed method was evaluated determining selected PACs in PM aerosol samples collected on quartz filters (at mass loadings of $0.2 \mathrm{mg}$ ) and $\mathrm{Al}$ foils (mass loadings of $0.02 \mathrm{mg}$ ).

From an analytical point of view, these samples can be considered as a worst extreme case: analytical methods have to allow the quantification of analyte in PM mass loadings of a few micrograms. Figure 2 corresponds to representative chromatograms obtained from the analyses of final extract volumes of $100 \mu \mathrm{L}$. It should be noted that the good resolution of chromatograms was obtained without the need of any additional step for clean-up. No selected NPAHs were detected in this kind of samples. The sum of PAH masses reached $75 \mathrm{ng}$ in quartz filter samples, while those for Al foils were only up to $2 \mathrm{ng}$. Regarding PM collected on Al foils, very slight differences were obtained between samples, with most of the compounds being below $0.1 \mathrm{ng}$. All the measurements remained slightly above the quantified limits established, so we considered these levels as detectable, not for quantitative purposes. Table 4 includes selected PAH measurements (ng) from the analyses of quartz filters. As can be seen, measured 


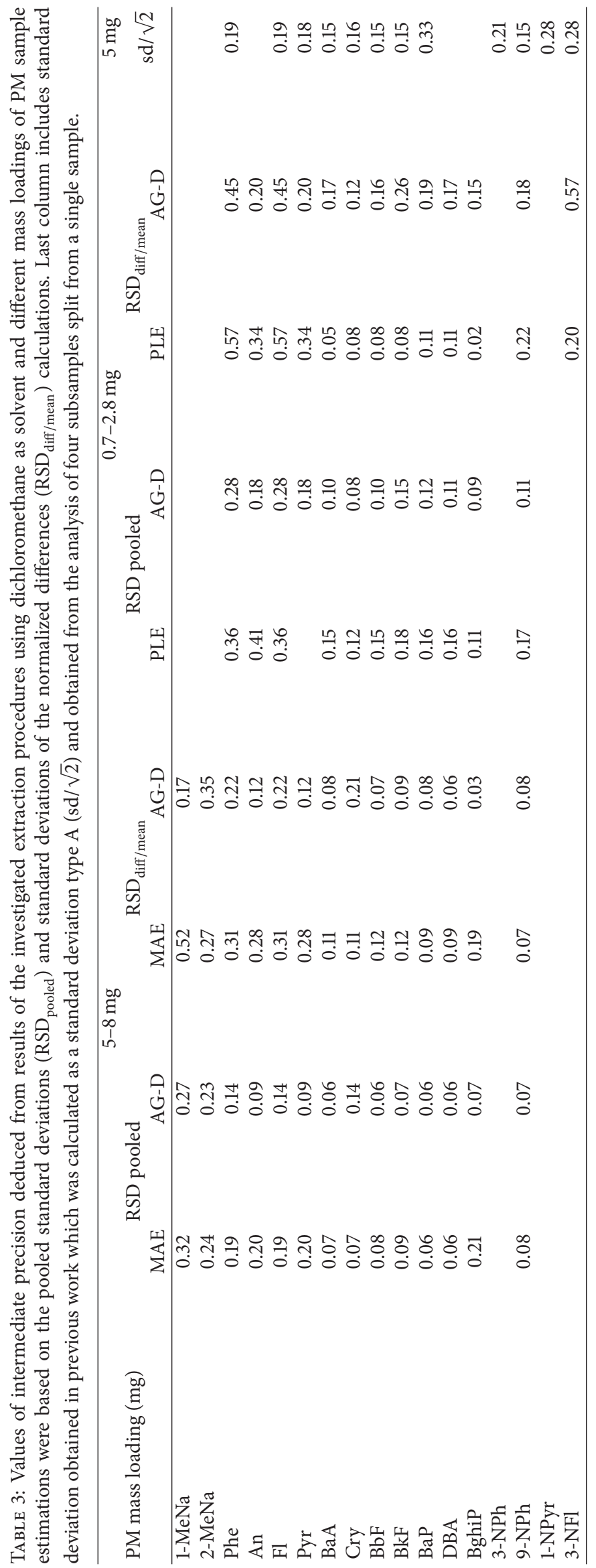




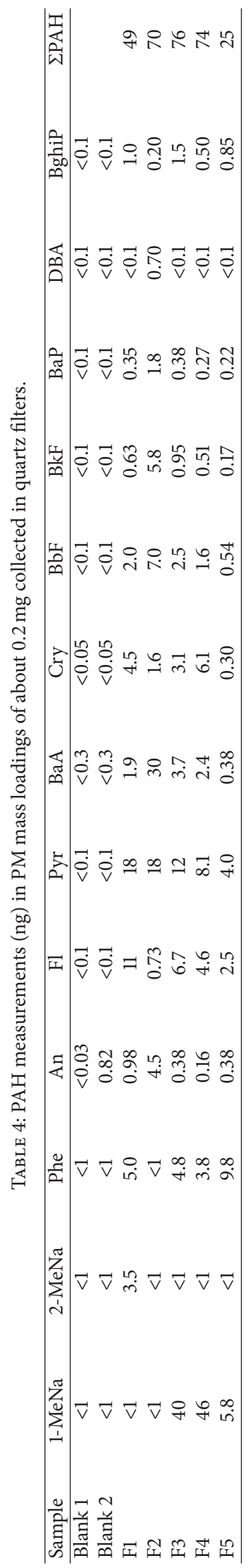




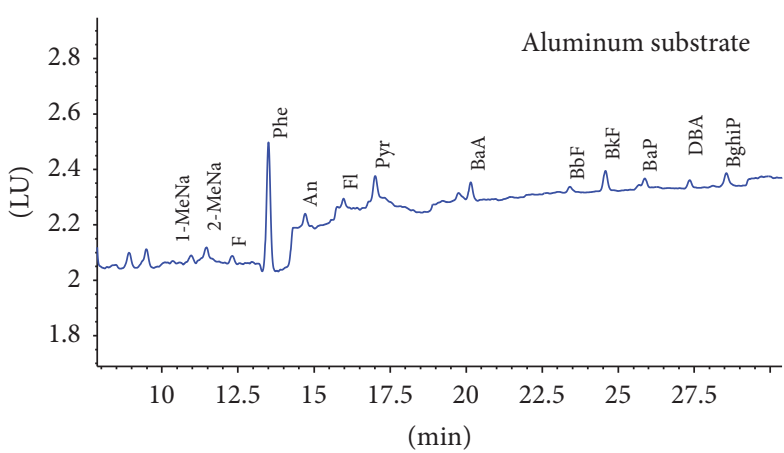

(a)



(b)

Figure 2: Chromatograms of samples collected on aluminum (a) and quartz (b) substrates at very low concentration levels. The PAH abbreviations are expanded in Reagents and Standards.

levels reached orders of units of nanogram, expressed as mass per analyzed filter.

Due to the lack of analytical assessments in this extremely low range of mass loadings, the real extent of this burden remains to be determined in future researches. Therefore, these preliminary results need additional studies on the characterization of size distribution from aircraft aerosol emissions. These goals are the main objectives to continue further studies from aircraft aerosol emissions.

\section{Conclusions}

We outlined the scope of a sonication/agitation extraction method with minimal handling and reagents for PAC determination in PM samples with low mass loadings $(<10 \mathrm{mg})$. Quality assessment of results was investigated from analysis of real samples. Therefore, we can conclude the following:

(i) The proposed method has been tested by comparison with extractions performed by microwave and pressurized solvent from real samples with PM mass loadings of $10 \mathrm{mg}$. In general, ratios between results obtained from the proposed method and PLE were above $80 \%$ for the most abundant PAHs and dichloromethane as solvent. Determination of selected NPAHs requires the use of methanol as modifier in extraction solvent to improve extraction efficiency.

(ii) Evaluation of intermediate precision was carried out by using two practical approaches. Both were in agreement. From analyses of samples with mass loadings between 0.7 and $8 \mathrm{mg}$, precision of around $10 \%$ for the majority of investigated PAHs was found. Precision close to $20 \%$ was found in the case of the most volatile PAHs and NPAHs.

(iii) The effectiveness of the method was evaluated to analyze samples with both very low mass loadings $(<0.2 \mathrm{mg})$ and concentration levels (a few ng in mass), that is, impactor substrates used to determine the nano- and submicron size distribution.

\section{Acronyms}

PM: $\quad$ Particulate matter

PACs: Polycyclic aromatic compounds

PAHs: Polycyclic aromatic hydrocarbons

NPAHs: Nitro polycyclic aromatic hydrocarbons

SX: $\quad$ Soxhlet

MAE: Microwave assisted extraction

PLE: $\quad$ Pressurized liquid extraction

US: Ultrasonic extractions

AG-D: Extraction by sonication/agitation using dichloromethane

AG-DM: Extraction by sonication/agitation using dichloromethane/methanol $2: 1$.

\section{Conflicts of Interest}

The authors declare that there are no conflicts of interest regarding the publication of this paper.

\section{Acknowledgments}

This work has been carried out in the framework of the INTA/CIEMAT research agreement.

\section{References}

[1] S. Percot, V. Ruban, P. Roupsard, D. Maro, and M. Millet, "Use of beryllium-7 as a surrogate to determine the deposition of metal and polycyclic aromatic hydrocarbon through urban aerosols in Nantes, France," Atmospheric Environment, vol. 74, pp. 338-345, 2013.

[2] Y. Zhu, L. Yang, Q. Yuan et al., "Airborne particulate polycyclic aromatic hydrocarbon (PAH) pollution in a background site in the North China Plain: Concentration, size distribution, toxicity and sources," Science of the Total Environment, vol. 466-467, pp. 357-368, 2014.

[3] M. Szewczyńska, M. Pośniak, and E. Dobrzyńska, "Study on individual PAHs content in ultrafine particles from solid fractions of diesel and biodiesel exhaust fumes," Journal of Chemistry, Article ID 528471, 2013. 
[4] A. I. Barrado, S. García, E. Barrado, and R. M. Pérez, "PM 2.5bound PAHs and hydroxy-PAHs in atmospheric aerosol samples: Correlations with season and with physical and chemical factors," Atmospheric Environment, vol. 49, pp. 224-232, 2012.

[5] L. Li-bin, Y. Yan, L. Jin-ming, T. Ning, and H. M. T. Kazuichi, "Development of analytical methods for polycyclic aromatic hydrocarbons (PAHs) in airborne particulates: a review," Journal of Environmental Sciences, vol. 19, no. 1, pp. 1-11, 2007.

[6] M. D. Hays, N. D. Smith, J. Kinsey, Y. Dong, and P. Kariher, "Polycyclic aromatic hydrocarbon size distributions in aerosols from appliances of residential wood combustion as determined by direct thermal desorption - GC/MS," Journal of Aerosol Science, vol. 34, no. 8, pp. 1061-1084, 2003.

[7] D. R. Anderson, M. J. Booth, P. Collins et al., "Ultrafine characterisation of emission and impact of ultrafine particulate," in EUR 23877 EN European Communities, 2009.

[8] R. T. Tremblay, D. D. Riemer, and R. G. Zika, "Organic composition of PM2.5 and size-segregated aerosols and their sources during the 2002 Bay Regional Atmospheric Chemistry Experiment (BRACE), Florida, USA," Atmospheric Environment, vol. 41, no. 20, pp. 4323-4335, 2007.

[9] L. Wolska, "Miniaturised analytical procedure of determining polycyclic aromatic hydrocarbons and polychlorinated biphenyls in bottom sediments," Journal of Chromatography A, vol. 959, no. 1-2, pp. 173-180, 2002.

[10] S. Ozcan, A. Tor, and M. E. Aydin, "Application of miniaturised ultrasonic extraction to the analysis of organochlorine pesticides in soil," Analytica Chimica Acta, vol. 640, no. 1-2, pp. 52-57, 2009.

[11] A. Christensen, C. Östman, and R. Westerholm, "Ultrasoundassisted extraction and on-line LC-GC-MS for determination of polycyclic aromatic hydrocarbons (PAH) in urban dust and diesel particulate matter," Analytical and Bioanalytical Chemistry, vol. 381, no. 6, pp. 1206-1216, 2005.

[12] A. Gustavo González and M. Ángeles Herrador, "A practical guide to analytical method validation, including measurement uncertainty and accuracy profiles," TrAC - Trends in Analytical Chemistry, vol. 26, no. 3, pp. 227-238, 2007.

[13] A. G. González, M. A. Herrador, and A. G. Asuero, "Intralaboratory testing of method accuracy from recovery assays," Talanta, vol. 48, no. 3, pp. 729-736, 1999.

[14] A. I. Barrado-Olmedo, R. M. Pérez-Pastor, and S. GarcíaAlonso, "An evaluation of uncertainty associated to analytical measurements of selected polycyclic aromatic compounds in ambient air," Talanta, vol. 101, pp. 428-434, 2012.

[15] T. Tomić, N. U. Nasipak, and S. Babić, "Estimating measurement uncertainty in high-performance liquid chromatography methods," Accreditation and Quality Assurance, vol. 17, no. 3, pp. 291300, 2012.

[16] M. Rynö, L. Rantanen, E. Papaioannou, A. G. Konstandopoulos, T. Koskentalo, and K. Savela, "Comparison of pressurized fluid extraction, Soxhlet extraction and sonication for the determination of polycyclic aromatic hydrocarbons in urban air and diesel exhaust particulate matter," Journal of Environmental Monitoring, vol. 8, no. 4, pp. 488-493, 2006.

[17] S. B. Hawthorne, C. B. Grabanski, E. Martin, and D. J. Miller, "Comparisons of Soxhlet extraction, pressurized liquid extraction, supercritical fluid extraction and subcritical water extraction for environmental solids: recovery, selectivity and effects on sample matrix," Journal of Chromatography A, vol. 892, no. 1-2, pp. 421-433, 2000.
[18] "Eurachem/CITAC Guide CG4 (2012) Quantifying Uncertainty in Analytical Measurement," http://www.eurachem.org.

[19] M. Piñeiro-Iglesias, P. López-Mahía, E. Vázquez-Blanco, S. Muniategui-Lorenzo, and D. Prada-Rodríguez, "Problems in the extraction of polycyclic aromatic hydrocarbons from diesel particulate matter," Polycyclic Aromatic Compounds, vol. 22, no. 2, pp. 129-146, 2002.

[20] V. Archilla, J. Rodríguez-Maroto, M. Izquierdo et al., "Characterisation of particulate bound PAHs emitted from aircraft turbofan engines," in Proceedings of the European Aerosol Conference, 2015. 

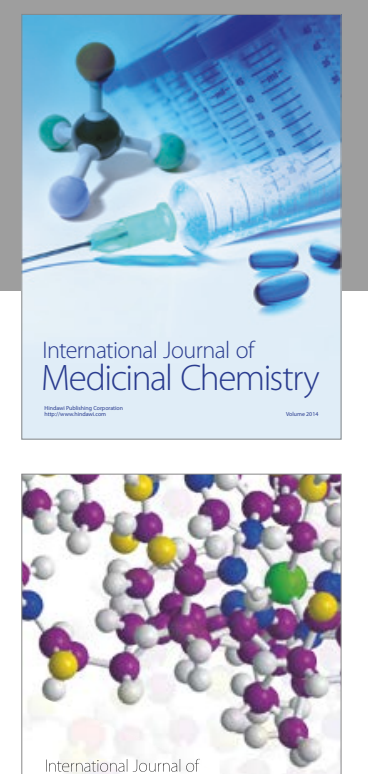

Carbohydrate Chemistry

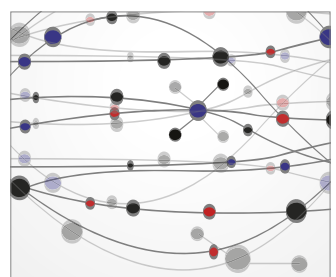

The Scientific World Journal
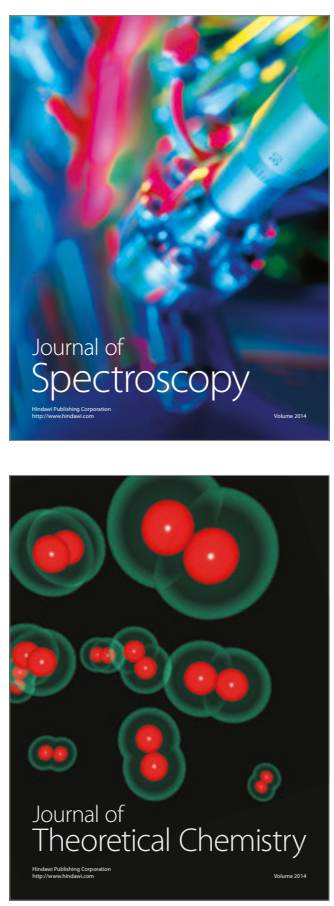
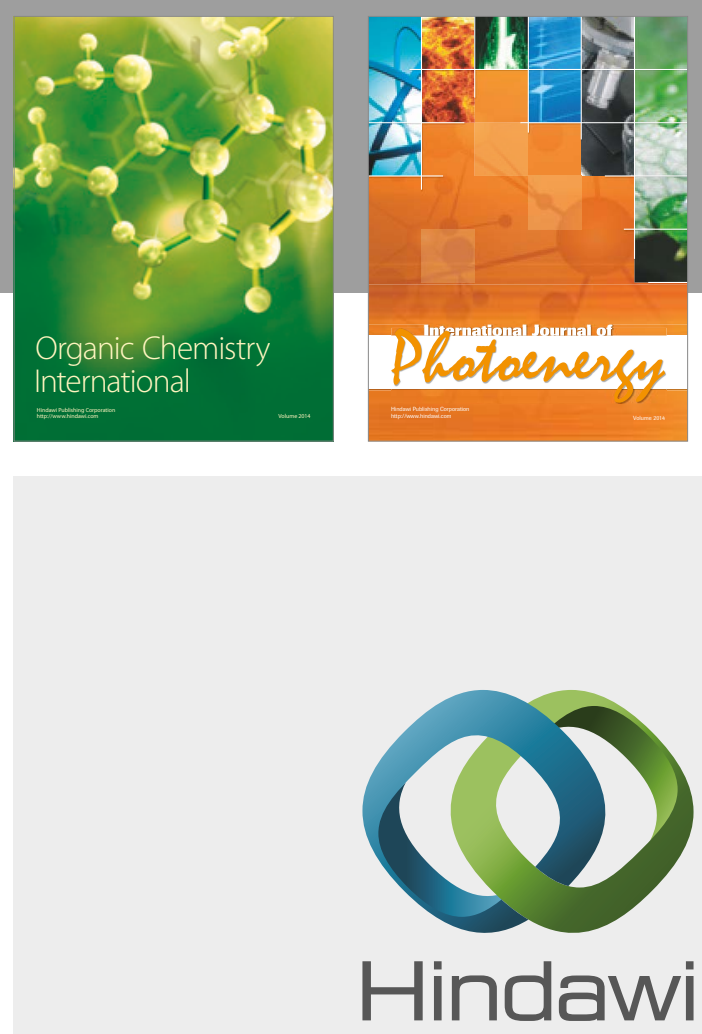

Submit your manuscripts at

https://www.hindawi.com

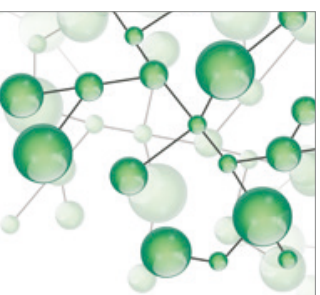

International Journal of

Inorganic Chemistry





Applied Chemistry
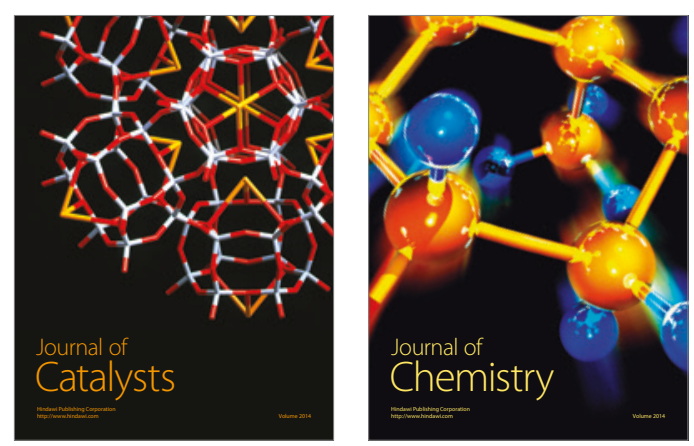
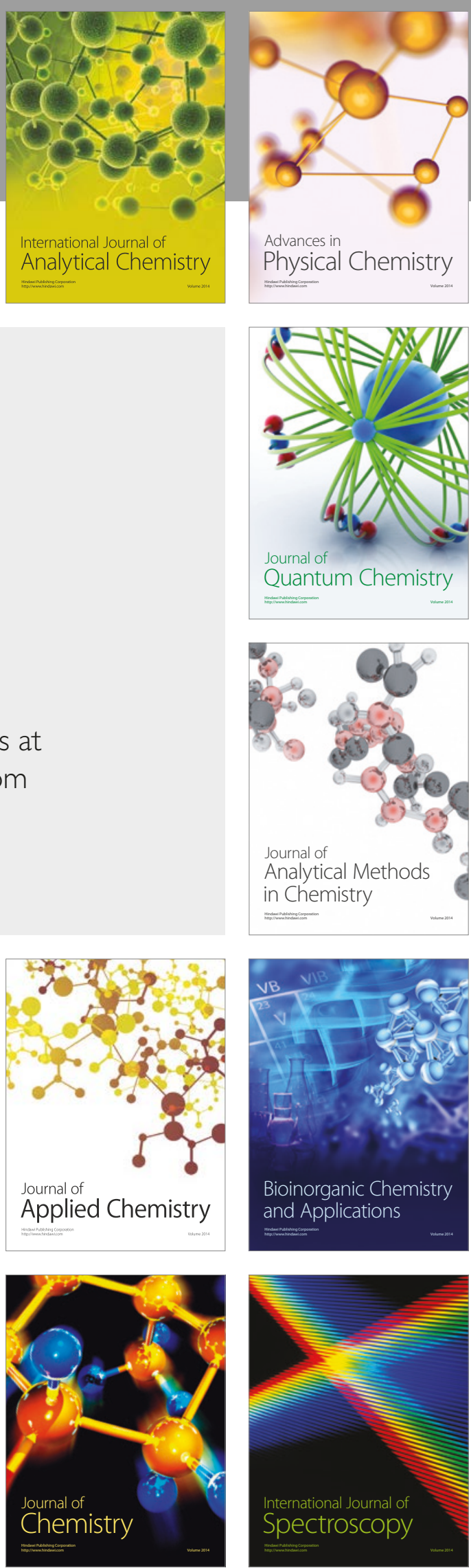